Published in "Complementary Therapies in Medicine 40(): 179-184, 2018"

which should be cited to refer to this work.

\title{
Acceptance, satisfaction and cost of an integrative anthroposophic program for pediatric respiratory diseases in a Swiss teaching hospital: An implementation report
}

\author{
Tido von Schoen-Angerer ${ }^{\mathrm{a}, \mathrm{b}, *}$, Jan Vagedes ${ }^{\mathrm{b}, \mathrm{e}}$, Romy Schneider ${ }^{\mathrm{a}}$, Livia Vlach ${ }^{\mathrm{a}}$, Cosette Pharisa ${ }^{\mathrm{a}}$, \\ Simon Kleeb ${ }^{\mathrm{c}}$, Johannes Wildhaber ${ }^{\mathrm{a}, \mathrm{d}}$, Benedikt M. Huber ${ }^{\mathrm{a}}$ \\ a Department of Pediatrics, Fribourg Hospital HFR, Fribourg, Switzerland \\ ${ }^{\mathrm{b}}$ ARCIM Institute, Filderklinik, Filderstadt, Germany \\ c Institute of Hospital Pharmacy, Fribourg Hospital HFR, Switzerland \\ ${ }^{\mathrm{d}}$ Department of Medicine, University of Fribourg, Switzerland \\ e Department of Pediatrics, University Hospital Tübingen, Germany
}

\section{A R T I C L E I N F O}

\section{Keywords:}

Integrative medicine

Complementary medicine

Anthroposophic medicine

Anthroposophy

Pediatrics

Bronchiolitis

Asthma

Pneumonia

Health care quality

Access

Evaluation

\begin{abstract}
A B S T R A C T
Background: For the pilot phase of an integrative pediatric program, we defined inpatient treatment algorithms for bronchiolitis, asthma and pneumonia, using medications and nursing techniques from anthroposophic medicine (AM). Parents could choose AM treatment as add-on to conventional care.

Material and methods: To evaluate the 18-month pilot phase, parents of AM users were asked to complete the Client Satisfaction Questionnaire (CSQ-8) and a questionnaire on the AM treatment. Staff feedback was obtained through an open-ended questionnaire. Economic data for project set-up, medications and insurance reimbursements were collected.

Results: A total of 351 children with bronchiolitis, asthma and pneumonia were hospitalized. Of these, 137 children (39\%) received AM treatment, with use increasing over time. 52 parents completed the questionnaire. Mean CSQ-8 score was 29.77 (95\% CI 29.04-30.5) which is high in literature comparison. 96\% of parents were mostly or very satisfied with AM; $96 \%$ considered AM as somewhat or very helpful for their child; $94 \%$ considered they learnt skills to better care for their child. The staff questionnaire revealed positive points about enlarged care offer, closer contact with the child, more relaxed children and greater role for parents; weak points included insufficient knowledge of AM and additional nursing time needed. Cost for staff training and medications were nearly compensated by AM related insurance reimbursements.

Conclusions: Introduction of anthroposophic treatments were well-accepted and led to high parent satisfaction. Additional insurance reimbursements outweighed costs. The program has now been expanded into a center for integrative pediatrics.
\end{abstract}

\section{Introduction}

Integrative medicine has increasing relevance and acceptance in pediatrics. ${ }^{1,2}$ Despite its popularity with parents there are however few integrative pediatric inpatient services available in Europe. ${ }^{2,3}$

The different available definitions of integrative medicine generally include notions of a holistic approach to the individual in its individual context, integration of complementary with conventional therapies, and patient-centered inter-professional collaboration. ${ }^{1,4}$

Different models of integrative health care service level have been described. $^{5-7}$
Mann et al., for example, have contrasted between conventional medicine practitioners that obtain complementary medicine training with multidisciplinary and interdisciplinary teams consisting of conventional and complementary medicine practitioners. ${ }^{6}$ Templeman et al. have differentiated between the selective integration of the most effective complementary and conventional methods (based on respect of the ontological differences between different schools of medicine), and the selective incorporation of evidence-based complementary medicine interventions.

As the first pediatric inpatient department in Switzerland, we wanted to provide integrative pediatric services within a public

* Corresponding author at: Dept of Pediatrics, Fribourg Hospital HFR, Chemin des Pensionnats 2, 1708 Fribourg, Switzerland.

E-mail address: tido.von.schoenangerer@gmail.com (T. von Schoen-Angerer). 
hospital, in response to the interest of families and interest within the nursing and physician team. Our aim was to provide an expanded and more holistic treatment offer and to further improve patient/family experience and outcomes. Our approach was to train the existing team of nurses and physicians in complementary medicine; to select one complementary medical system - anthroposophic medicine - for integration rather than selectively incorporating individual therapies from different complementary systems; and to begin the integrative approach for patients with selected, conventionally defined pathologies.

Anthroposophic medicine is an integrative medical system using multimodal treatment concepts based on a holistic understanding of man and nature. It uses medicines based on plants, minerals and animals, and different body therapies including specific nursing techniques such as compresses and massages. ${ }^{9}$ Anthroposophic medicine is always practiced in integration with conventional medicine.

Switzerland provides a favorable context for integrative medicine following a national referendum in favor of integrating complementary medicine into regular health services. As a result, there are recognized post-graduate physician degrees for selected complementary systems and reimbursement for complementary treatments through the compulsory, basic insurance scheme. ${ }^{10,11}$

We here provide a report on the planning and implementation experience.

\section{Methods}

\subsection{Project planning and implementation}

The pediatrics department at Fribourg hospital serves the canton of Fribourg and includes a general pediatrics and a neonatology ward with a total of 24 beds. We opted to begin with a pilot phase of adding complementary treatments for children hospitalized for bronchiolitis, asthma/obstructive bronchitis and pneumonia. Respiratory diseases were selected as these are common in pediatrics and because of the interest of the head of the department, a pediatric pulmonologist.

Among the different complementary medical systems we choose to employ anthroposophic medicine, as there is significant inpatient experience with this approach - including in pediatrics - and because one of the attending physicians was a certified provider in anthroposophic medicine.

A project coordination group was established including two attending pediatricians, one neonatal nurse and one nurse from the general pediatric unit. No new staff positions were created and the project received no external funding. The project was proposed to the hospital director where it received full support. A pediatric department of an integrative hospital with four decades of experience in integrating anthroposophic medicine (Filderklinik, Germany) was identified as partner for technical support.

It was decided to establish a standardized treatment protocol so that integrative treatments could be provided at any time of day and night, and by physicians and nurses without expert knowledge in anthroposophic medicine.

The anthroposophic treatment protocol was established in collaboration with a pediatric expert from our partner, the Filderklinik, based on anthroposophic medical literature and experience at the Filderklinik. ${ }^{12-17}$ The protocol included inhalations, oral medications and nursing applications (see Fig. 1). Medications included preparations from plants, minerals and animals, principally from the manufacturers WELEDA (Schwäbisch Gmünd, Germany) and WALA (Bad Boll, Germany) and were obtained through the hospital pharmacy; all WALA and WELEDA medications had registrations with Swissmedic, the Swiss national regulatory agency. A detailed nursing protocol was developed for all the nursing methods (general instructions on anthroposophic nursing applications are available at http://www.pflegevademecum.de/[accessed October 18, 2017]).
Physicians and nursing staff were trained separately to apply the protocol. Physicians received a 2 -h introduction into anthroposophic medicine with instructions how to apply the protocol by the project coordinator. Nurses received a 1-day training on anthroposophic nursing techniques by an external nursing expert (training was repeated on three occasions to ensure participation of all nurses). Nurses received a half-day training refresher 10 months into the project. Physicians had ongoing support available through the attending physician certified in anthroposophic medicine.

A flyer for parents informing about the project and the option of complementary therapy was handed out on hospital admission; a more detailed booklet about anthroposophic medicine was available on request. Private practice pediatricians in the canton of Fribourg were informed about the project through the local pediatric society. Four local pharmacies were requested to hold the medications on stock that children would need after discharge.

Implementation of the complementary treatment was begun in January 2015 for a pilot phase of 18 months. On hospital admissions for bronchiolitis, asthma and pneumonia parents were offered the complementary treatment as an optional add-on to conventional care. During hospitalization parents were taught how to apply chest compresses and massages and were encouraged to actively participate in the nursing care.

\subsection{Project evaluation}

An evaluation of the pilot phase from January 2015 through June 2016 was part of the project management to understand acceptance of and satisfaction with the integrative treatment program.

Number of hospital admissions for bronchiolitis, asthma and pneumonia and number of patients receiving the complementary care option were calculated from hospital records.

Parents of children receiving complementary treatment were asked to complete two questionnaires on hospital discharge. First, the Client Satisfaction Questionnaire CSQ-8 (CSQ-8 French version, (C) Clifford Attkisson 2012; ZUF-8 German version, (C) J. Schmidt et al., 1989, 1994) which has an internal consistency Cronbach alpha of $0.83-0.93 .^{19,28}$ Minor language changes were made to the CSQ-8 to improve French grammar, comprehensibility as well as consistency with the German version (Fribourg hospital is a bilingual French \& German institution). For the CSQ-8/ZUF-8 the arithmetic total score was calculated. ${ }^{18}$ Secondly, we used a questionnaire on complementary treatment satisfaction that was developed and pre-tested in-house, using a 4-scale responses, e.g. "very satisfied", "somewhat satisfied", "somewhat dissatisfied", "dissatisfied" (see Fig. 3 for questions).

Physicians and nurses were asked to complete an open-ended questionnaire at 6 months into the pilot phase. Questions were asked about general impressions about the complementary treatments, sufficiency of information/training received, advantages/disadvantages in daily practice, observed effects, parent reactions and suggestions.

A cost-minimization analysis was carried out from a hospital perspective $^{20}$ : direct expenses for training and anthroposophic medicines were collected. Staff cost was not included as no additional staff time was paid for. Additional DRG-based hospital reimbursements for complementary medicine were measured. Given limited data on the efficacy of the complementary treatments provided we assumed equal outcomes with and without complementary treatment for the purpose of the costminimization analysis.

The project complied with the principles of the Helsinki declaration. No ethics approval was sought as this was an implementation of known, approved treatments. The evaluation was an integral part of the project management cycle. 


\begin{tabular}{|c|c|c|c|}
\hline Administration route & Product / treatment & Ingredients (if multiple in product) & Frequency \\
\hline \multicolumn{4}{|c|}{ Indication: Bronchiolitis } \\
\hline Oral & Stibium arsenicosum D8 & & 3 times daily \\
\hline Inhalation & Pulmo/Vivianit comp. & $\begin{array}{l}\text { Pulmo bovis D16, Tararus stibiatus D7, } \\
\text { Vivianit D7 }\end{array}$ & Twice daily \\
\hline Inhalation & Apis D10 + Quarz D12 & & $\begin{array}{l}\text { Twice daily, alternating } \\
\text { with Pulmo/Vivianit }\end{array}$ \\
\hline Nursing application & Chest compress with thyme tea & & In the morning \\
\hline Nursing application & $\begin{array}{l}\text { Chest compress with lavender oil } \\
10 \%\end{array}$ & & In the evening \\
\hline \multicolumn{4}{|l|}{ Indication: Asthma } \\
\hline Oral & $\begin{array}{l}\text { Cuprum metallicum } \\
\text { praeparatum D10 }\end{array}$ & & 3 times daily \\
\hline Inhalation & Cuprum aceticum comp. & $\begin{array}{l}\text { Cuprum aceticum D5, Nicotiana tabacum } \\
\text { D9, Renes bovis D5 }\end{array}$ & 4 times daily \\
\hline Inhalation & Nicotiana comp. & $\begin{array}{l}\text { Carbo vegetabilis D19, Chamomilla recutita } \\
\text { e radice } D 2 \text {, Nicotiana tabacum D9 }\end{array}$ & $\begin{array}{l}4 \text { times daily, with Cuprum } \\
\text { aceticum comp }\end{array}$ \\
\hline Inhalation & Gencydo $^{\circ} 1 \%+$ Quarz D12 & Gencydo $^{\oplus}$ : Citrus limon, Cydonia oblonga & $\begin{array}{l}\text { Alternative to Cuprum } \\
\text { aceticum/Nicotiana, if } \\
\text { known allergic trigger }\end{array}$ \\
\hline Nursing application & $\begin{array}{l}\text { Chest compress with } \\
\text { Retterspitz }\end{array}$ & $\begin{array}{l}\text { Essential oils of lemon, rosemary, thyme, } \\
\text { bergamot, orange flour; Arnica } \emptyset\end{array}$ & Twice daily \\
\hline Nursing application & $\begin{array}{l}\text { Food baths prepared with ginger } \\
\text { powder }\end{array}$ & & In the evening \\
\hline Nursing application & $\begin{array}{l}\text { Foot and calf massage with } \\
\text { Cuprum metallicum } 0.4 \% \\
\text { ointment }\end{array}$ & & After foot bath \\
\hline \multicolumn{4}{|c|}{ Indication: Pneumonia - $1^{\text {st }}$ week } \\
\hline Oral & $\begin{array}{l}\text { Aconitum napellus D6 + Bryonia } \\
\text { D6 + Phosphor D5/Tartarus } \\
\text { stibiatus D3 + } \\
\text { Ferrum phosphoricum D6 }\end{array}$ & & 3 times daily \\
\hline Inhalation & Pulmo/Vivianit comp & $\begin{array}{l}\text { Pulmo bovis D16, Tararus stibiatus D7, } \\
\text { Vivianit D7 }\end{array}$ & 3 times daily \\
\hline Nursing application & Chest compress with ginger & & In the morning \\
\hline Nursing application & $\begin{array}{l}\text { Chest compress with lavender oil } \\
10 \%\end{array}$ & & In the evening \\
\hline \multicolumn{4}{|c|}{ Indication: Pneumonia $-2^{\text {nd }}$ week and after discharge } \\
\hline Oral & Ferrum sidereum D6 & & 3 times daily \\
\hline Inhalation & Pulmo/Vivianit comp & $\begin{array}{l}\text { Pulmo bovis D16, Tararus stibiatus D7, } \\
\text { Vivianit D7 }\end{array}$ & 3 times daily \\
\hline Nursing application & $\begin{array}{l}\text { Chest compress with "Plantago } \\
\text { Bronchialbalsam" } \\
\text { (minimum age } 1 \text { year) }\end{array}$ & $\begin{array}{l}\text { D-Campher, Cera flava, Eucalypti } \\
\text { aetheroleum, Petasites, Plantago } \\
\text { lanceolata, Terebinthina laricina, Thymi } \\
\text { aetheroleum }\end{array}$ & During day time \\
\hline Nursing application & $\begin{array}{l}\text { Chest compress with lavender oil } \\
10 \%\end{array}$ & & At night \\
\hline \multicolumn{4}{|c|}{ Indication: Fever management (to be tried for 1 hour, if still inadequate comfort acetaminophen or ibuprofen) } \\
\hline Rectal & Chamomilla comp. & $\begin{array}{l}\text { Argentum metallicum D19, Atropa } \\
\text { belladonna D3, Chamomilla D2, Echinacea } \\
\text { angustifolia } \varnothing \text {, Echinacea purpurea } \emptyset \text {, } \\
\text { Papaver somniferum D3 }\end{array}$ & Up to 3 times daily \\
\hline Rectal & $\begin{array}{l}\text { Echinacea / Mercurius comp. } \\
\text { (alternative to Chamomilla } \\
\text { comp, if adenitis or pharyngitis) }\end{array}$ & $\begin{array}{l}\text { Apis mellifica D3, Argentum metallicum } \\
\text { D18, Atropa belladonna D2, Echinacea } \varnothing \text {, } \\
\text { Mercurius solubilis Hahnemanni D13 }\end{array}$ & Up to 3 times daily \\
\hline Nursing application & $\begin{array}{l}\text { Calf compresses with tepid } \\
\text { lemon water }\end{array}$ & & Up to 4 times daily \\
\hline
\end{tabular}

Fig. 1. Anthroposophic treatment protocol.

All treatments provided in addition to conventional treatment. $\emptyset=$ mother tincture; $\mathrm{D}=$ denotes decimal dilution, e.g. D8 means $1: 10^{8}$.

\section{Results}

\subsection{Treatment uptake}

A total of 351 children with bronchiolitis, asthma and pneumonia were hospitalized during the 18-month pilot phase. Of these 351 children, 136 children (39\%) received complementary treatment. Broken down by disease, $51 / 115$ (44\%) of children with bronchiolitis, $27 / 113$
(24\%) with asthma, 58/123 (47\%) with pneumonia and 2 with other respiratory diagnosis received the complementary treatment. The use of anthroposophic treatment increased over time (see Fig. 2).

\subsection{Patient satisfaction}

Parents of 52 children receiving complementary treatment completed the questionnaires, 27 (54\%) of these had never before used 




Fig. 2. Uptake of complementary treatment over time. $\%$ of all children hospitalized for bronchiolitis, asthma or pneumonia receiving complementary treatment.

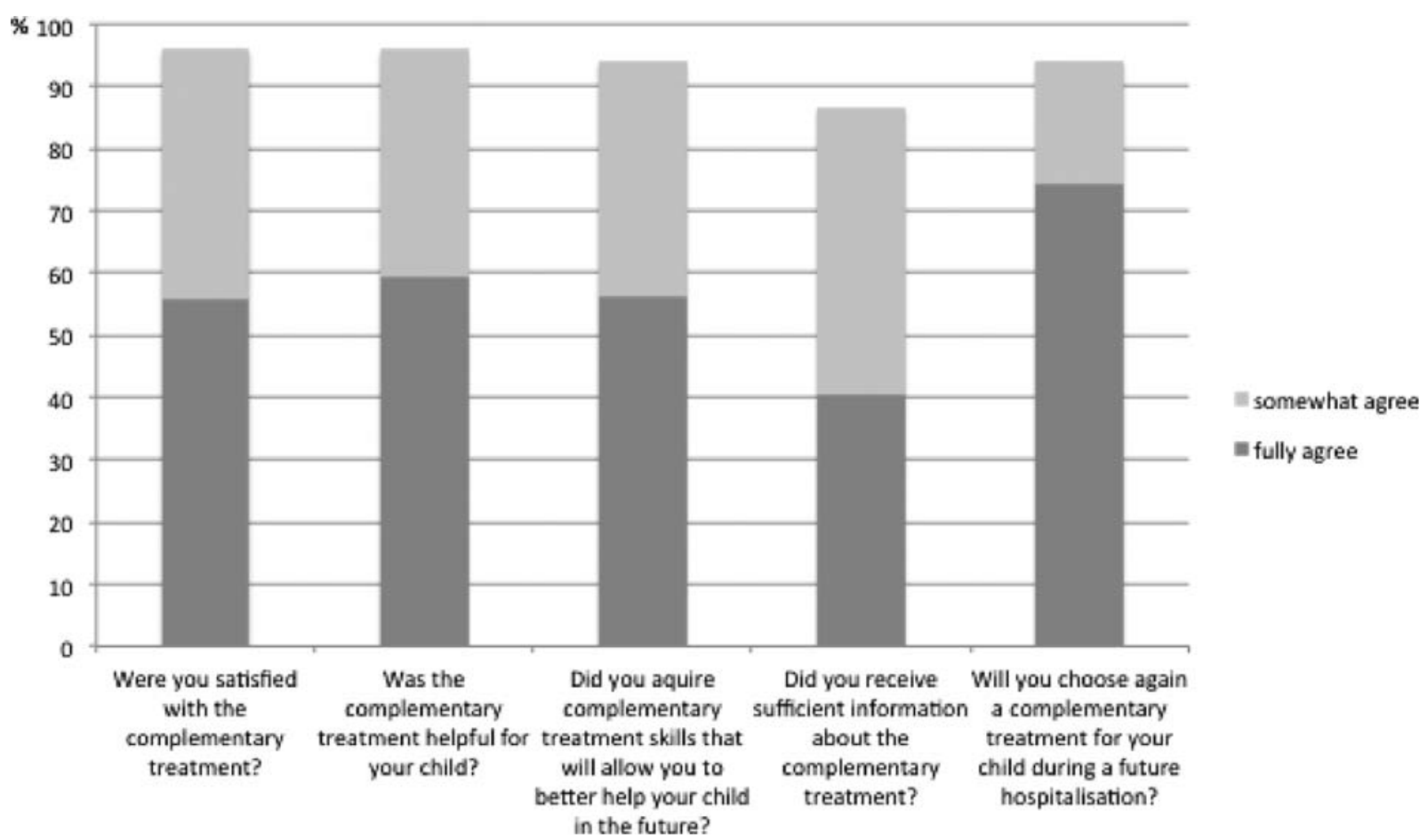

Fig. 3. Parent responses to questions about the complementary treatment.

complementary medicine for their child. The most common, previously used complementary medicine was homeopathy.

Mean CSQ-8 score was 29.77 (95\% CI 29.04-30.5) out of a possible maximum score of 32 .

$96 \%$ of parents were mostly or very satisfied with the complementary treatment; $96 \%$ considered it as somewhat or very helpful for their child; $94 \%$ considered they learnt care skills to better care for their child in the future; $87 \%$ thought they received sufficient information about the complementary treatment (see Fig. 3).

\subsection{Staff feedback and satisfaction}

6 physicians and 43 nurses filled the staff questionnaire.

Physicians noted positively: the additional treatment offer; active parent involvement in the care; a more human approach in the care, and a more listening and close contact with the child and parents. They felt children tolerated the treatments well but that the various different inhalations were difficult to comply with. Physicians also wanted to better understand the basic concepts of anthroposophic medicine and the mechanisms of actions of the treatments. Several respondents suggested expanding the complementary treatment offer to other pathologies.

Nurses noted positively: the additional treatment offer; a closer, comforting contact with the child; moments of relaxation between child and parents; active participation by parents; and satisfaction and pleasure from performing concrete care activities. Negative comments included the additional time needed to prepare and perform the nursing applications; inadequate space for storage of the material and for preparation of the interventions; and frustration if after preparation a child refused the intervention. Nurses noted difficulty to apply various complementary and conventional inhalations (in response it was agreed that conventional and complementary aerosols could be administered together). Many nurses noted they still had too little experience with the new nursing techniques at the time of the questionnaire. Nurses observed that the nursing applications led to reduced cough, improved respiration, calmer night sleep and relaxation. Two types of parent categories were observed: "the skeptics" and those open and even enthusiastic about the complementary treatment option.

\subsection{Cost-minimization analysis}

Expenses: 8.785 US\$ for training (cost for trainer) and 6.473 US\$ for anthroposophic medications (of this 411 US\$ for oral, 4.475 US\$ for inhalation, 187 US\$ for rectal and 1401 US\$ for external medications), totaling an additional cost for anthroposophic care of 15.258 US\$.

Additional DRG-based insurance reimbursements received for the 
complementary treatment(according to the Swiss DRG system complementary medicine treatment involving at least $10 \times 30$ min nursing interventions per hospitalization increases the DRG-based reimbursement): 15.044 US\$.

The additional cost for the department was nearly outweighed by additional insurance reimbursements (income - expenses $=-214$ US \$).

\section{Discussions and conclusions}

The introduction of complementary, anthroposophic treatments for respiratory disorders as part of an integrative medicine concept in a Swiss pediatric inpatient department was well accepted by patients and staff and led to high parent satisfaction. Start-up cost was nearly compensated by additional insurance reimbursements already during the pilot phase.

Acceptance and uptake by parents increased over time (Fig. 2) but we believe this was mostly due to increasing staff confidence and encouragement by the head of the department to systematically offer the new treatments.

Overall patient satisfaction (CSQ-8) was high in literature comparison: median scores between 26 and 27 have been report from childbirth related care, family and mental health care. ${ }^{21,22}$ As a limitation, CSQ-8 scores were not measured for children receiving conventional care only. The relatively low response rate to the questionnaire was mainly a result of failing to consistently distribute the questionnaire at the end of hospitalization rather than a lack of parental unwillingness to respond.

The positive feedback from our pediatric team was in line with observation at other centers where the integration of complementary medicine was perceived to improve holistic capacity through treating - the 'whole person', by filling gaps in existing service delivery, and through increasing treatment options for patients. ${ }^{23}$ In retrospect, we implemented the staff questionnaire too early when many nurses had little experience with the new techniques and could feel overwhelmed; more recent, informal feedback indicates ease with nursing interventions and less time constraints.

Both parent and staff responses have highlighted the need for more information about anthroposophic medicine which is based on a complex philosophy; providing more staff training and information material for patients has been agreed as a priority for future work.

The pilot phase provided a scaled-down and perhaps less effective version of anthroposophic medicine as treatment was not individualized and additional anthroposophic therapies, such as movement or art therapies could not be offered. Furthermore, the fever management protocol with focus on comfort rather than fever suppression was only partially implemented because it meant a culture change for the staff that we did not achieve during the pilot phase. Reduced fever suppression is a core element of the anthroposophic approach to infectious disease management ${ }^{24}$ and is in line with current evidence. $^{25}$

When considering lessons learnt, the following factors seem to have contributed to a successful project and might make it replicable elsewhere: strong support from the head of department and hospital direction; having a complementary medicine certified physician to champion the project and work with a coordination group; beginning small with a clear treatment protocol for selected pathologies. Similar factors of success have been identified by other hospitals starting to provide integrative medicine. ${ }^{26,27}$ Challenges included difficulty to familiarize an entire team with a complex complementary medical system such as anthroposophic medicine, introducing new nursing procedures on top of existing workload, and staff turnover. Three out of four members of the initial coordination group had left after the pilot phase; fortunately, excellent replacement was found. We were in the fortunate position that all staff was more or less open to the project and we did not face internal opposition. The favorable insurance reimbursement situation might be unique for Switzerland.

Following the successful pilot phase, a center for integrative pediatrics was created which includes an expanded offer of anthroposophic treatments also for other pathologies and an integrative pediatrics outpatient consultation. Public talks have been held to educate parents about integrative care approaches. Positive media attention was received through a report on Swiss public television. Prospective studies to increase the evidence of integrative treatment are under preparation.

Funding

None.

\section{Acknowledgements}

We thank Annette Beisswenger, expert in anthroposophic nursing and all the HFR pediatric staff for their enthusiasm and commitment in this project.

\section{References}

1. McClafferty H, Vohra S, Bailey M, et al. Pediatric integrative medicine. Pediatrics. 2017;140(3):e20171961.

2. Kemper KJ, Vohra S, Walls R, et al. The use of complementary and alternative medicine in pediatrics. Pediatrics. 2008;112(6):1374-1386.

3. Zuzak TJ, Boňková J, Careddu D, et al. Use of complementary and alternative medicine by children in Europe: published data and expert perspectives. Complement Ther Med. 2013;21(Suppl. 1):S34-S47.

4. Hu X-Y, Lorenc A, Kemper K, et al. Defining integrative medicine in narrative and systematic reviews: a suggested checklist for reporting. Eur J Integr Med. 2015;7(1):76-84.

5. Boon H, Verhoef M, O'Hara D, Findlay B. From parallel practice to integrative health care: a conceptual framework. BMC Health Serv Res. 2004:4.

6. Mann D, Gaylord S, Norton S. Moving toward integrative care: rationales, models and steps for conventional-care providers. Complement Health Pract Rev. 2004:9.

7. Leckridge B. The future of complementary and alternative medicine-models of integration. J Altern Complement Med. 2004;10(2):413-416.

8. Templeman K, Robinson A. Integrative medicine models in contemporary primary health care. Complement Ther Med. 2011;19(2):84-92

9. Kienle GS, Albonico H-U, Baars E, et al. Anthroposophic Medicine: an integrative medical system originating in Europe. Glob Adv Health Med. 2013;2(6):20-31.

10. Schweizerische_Eidgenossenschaft. Verordnung über die Krankenversicherung (KVV), Änderung vom 16. Juni 2017. Vol SR 832.102. 2017, 3687-3688.

11. Schweizerische_Eidgenossenschaft. Verordnung des EDI über Leistungen in der obligatorischen Krankenpflegeversicherung (Krankenpflege - Leistungsverordnung, KLV), Änderung vom 16. Juni 2017. Vol SR 832.112.31. 2017, 3689-3692.

12. Hamre HJ, Glockmann A, Schwarz R, et al. Antibiotic use in children with acute respiratory or ear infections: prospective observational comparison of anthroposophic and conventional treatment under routine primary care conditions. Evid Based Complement Altern Med. 2014;2014:243801.

13. Hamre HJ, Witt CM, Kienle GS, et al. Anthroposophic therapy for asthma: a two-year prospective cohort study in routine outpatient settings. J Asthma Allergy. 2009;2:111-128.

14. Hamre HJ, Glockmann A, Fischer M, et al. Use and safety of anthroposophic medications for acute respiratory and ear infections: a prospective cohort study. Drug Target Insights. 2007;2:209-219.

15. Soldner G, Stellman HM. Individual paediatrics: physical, emotional and spiritual aspects of diagnosis and counseling — anthroposophic-homeopathic therapy. 4th ed. MedPharm; 2014.

16. Schönau E, Naumann E, Längler A, Beuth J. Pädiatrie integrativ. München: Urban und Fischer; 2005.

17. Geyer U, Diederich $\mathrm{K}$, Kusserow M, et al. Inpatient treatment of community-acquired pneumonias with integrative medicine. Evid Based Complement Altern Med. 2013;2013:578274.

18. Attkisson CC, Greenfield T. The client satisfaction questionnaire (CSO) scales, outcome assessment in clinical practice. Baltimore, MD: Williams \& Wilkins; 1995:2-10.

19. Schmidt J, Wittmann WW. med Psychol VP, CSQ CSQ. Fragebogen zur Messung der Patientenzufriedenheit. Paper presented at: Diagnostische Verfahren in der Psychotherapie. Göttingen, Hogrefe2002; 2002.

20. Smith S, Sinclair D, Raine R. Health care evaluation. McGraw-Hill Education (UK); 2005.

21. Matsubara C, Green J, Astorga LT, et al. Reliability tests and validation tests of the client satisfaction questionnaire (CSQ-8) as an index of satisfaction with childbirthrelated care among Filipino women. BMC Pregnancy Childbirth. 2013;13(1):235.

22. Attkisson C, Greenfield T. The UCSF client satisfaction scales: I. The client satisfaction questionnaire-8. 3rd ed.In: Maruish ME, ed. The use of psychological testing for treatment planning and outcomes assessment. Vol. 3. Mahwah, NJ: Lawrence Erlbaum Associates; 2004. 
23. Singer J, Adams J. Integrating complementary and alternative medicine into mainstream healthcare services: the perspectives of health service managers. $B M C$ Complement Altern Med. 2014;14(1):1-11.

24. Martin DD. Fever: views in anthroposophic medicine and their scientific validity. Evid Based Complement Altern Med. 2016;2016:13.

25. Sullivan JE, Farrar HC. Fever and antipyretic use in children. Pediatrics. 2011;127(3):580-587.
26. Vohra S, Feldman K, Johnston B, et al. Integrating complementary and alternative medicine into academic medical centers: experience and perceptions of nine leading centers in North America. BMC Health Serv Res. 2005;5(1):1.

27. Boon H, Kachan N. Integrative medicine: a tale of two clinics. BMC Complement Altern Med. 2008:8

28. Attkisson C. Administering and scoring the CSQ scales. Mill Valley, CA: Tamalpais Matrix Systems LLC. 2012. 